\title{
A MMIC GaN HEMT Voltage-Controlled-Oscillator with high tuning linearity and low phase noise
}

\author{
Thanh Ngoc Thi Do ${ }^{\#}$, Szhau Lai ${ }^{\#}$, Mikael Hörberg ${ }^{\#}$, Herbert Zirath ${ }^{\#}$, Dan Kuylenstierna ${ }^{\#}$ \\ "Microwave Electronics Laboratory, Department of Microtechnology and Nanoscience, Chalmers \\ University of Technology, SE-42196, Gothenburg, Sweden.
}

\begin{abstract}
This paper presents a MMIC GaN HEMT Voltage-Controlled-Oscillator (VCO). The VCO is tunable between 6.45-7.55 GHz with good tuning linearity, average output power about $1 \mathrm{dBm}$, and a good phase noise with little variation over the tuning range. For a bias of $V_{d} / I_{d}=6 \mathrm{~V} / 33 \mathrm{~mA}$, the measured phase noise is $-98 \mathrm{dBc} / \mathrm{Hz} @$ $100 \mathrm{kHz}$ and $-132 \mathrm{dBc} / \mathrm{Hz} @ 1 \mathrm{MHz}$ offset frequencies, respectively. To the author's best knowledge, this is the lowest phase noise reported for a VCO in GaN HEMT technology with comparable oscillation frequency and tuning range. The $1 \mathrm{MHz}$ offset phase noise is also comparable to state-of-the-art GaAs-InGaP HBT VCOs with similar tuning range.
\end{abstract}

Index Terms - Voltage-Controlled-Oscillator, MMIC, GaN, HEMT, phase noise.

\section{INTRODUCTION}

There is a need for high purity signal generation in modern wireless communication systems and radar systems. In the systems using advanced modulation formats, e.g., higher order QAM, the phase noise of the voltage-controlled-oscillator (VCO) is one of the bottle necks impinging system performance [1].

A key property for design of low phase noise oscillators is the power handling capability of the active device. A transistor technology with particularly high breakdown voltage is GaN HEMT. A VCO based on GaN HEMT technology can theoretically reach very low phase noise. A number of GaN HEMT VCOs have been reported [2-4], but none of them present performances near the theoretical limit, and in particular the performances reported are yet not comparable to other technologies such as GaAs-InGaP HBT.

A major challenge in the design of low-phase noise GaN HEMT oscillators is the relatively high flicker noise which is up-converted to phase noise around the microwave carrier [5]. However, it is recently demonstrated for fixed-frequency GaN HEMT oscillators, that fairly low phase noise can be reached if the resonator impedance is well matched to the active device and the bias condition is carefully selected to avoid extensive flicker noise problems [6-7].

In this paper, a medium bandwidth GaN HEMT MMIC VCO with good phase noise and fairly linear tuning is demonstrated. The VCO is designed based on a balanced Colpitts topology. It has a tuning range of more than $15 \%$ over a frequency band from $6.45-7.55 \mathrm{GHz}$. The achieved phase noise at $1 \mathrm{MHz}$ offset is better than $-130 \mathrm{dBc} / \mathrm{Hz}$ over the tuning range which is comparable to the performance of GaAs-InGaP HBT VCOs.

\section{OSCILLATOR DESIGN}

The oscillator was designed and fabricated in UMS's GH25-10 GaN MMIC HEMT process, with a $f_{\mathrm{T}} \approx 30 \mathrm{GHz}$. In Figs. 1(a) and 1(b), respectively, the schematic and chip photo of the designed VCO are shown.

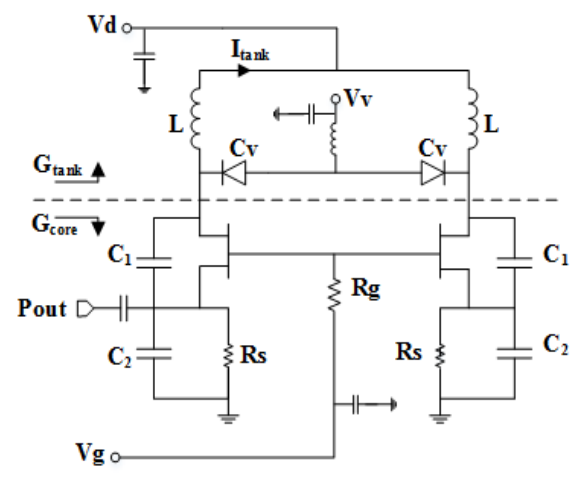

(a)

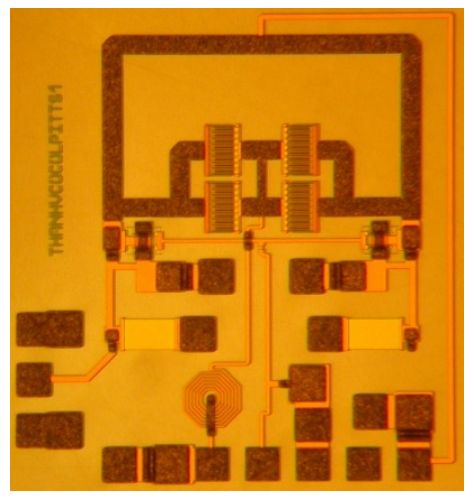

(b)

Fig. 1. Balanced Colpitts GaN HEMT VCO. (a) Schematic. (b) Chip photo. 
The common gate balanced Colpitts topology is chosen since it is known for low up-conversion of flicker noise. Some experimental work have also shown that the balanced Colpitts topology provides low phase noise for MMIC oscillators [8] and it is suitable for wideband tuning [9]. The transistor size is $2 \times 50 \mu \mathrm{m}$. The component values in the schematic are chosen for optimum phase noise. The tapping ratio of the capacitive divider in Fig. 1(a) $\left(n=C_{1} /\left(C_{1}+C_{2}\right)\right)$ is optimized to 0.3 . The width of the microstrip line in the tank inductance is $50 \mu \mathrm{m}$. A varactor size of $8 \times 50 \mu \mathrm{m}$ is chosen for wide tuning range. Due to the limitation of varactor dimensions, two varactors are combined in parallel in order to reach the desired capacitance value. The gate voltage is injected through a resistance of $1 \mathrm{k} \Omega$ while the value of the source resistance is $R_{s}=50 \Omega$. The output signal is extracted from a small MIM capacitance of $0.2 \mathrm{pF}$ at one RF port while the other RF port is open. The size of the $\mathrm{VCO}$ is $1300 \times 1400 \mu \mathrm{m}^{2}$.

The circuit is designed with Agilent Advanced Design system (ADS 2009). Time-variant phase noise predictions are based on waveform analysis in ADS harmonic balance (HB) and a low-frequency noise model according to the method presented in [10].

\section{EXPERIMENTAL RESULT}

\section{A. Varactor characterization}

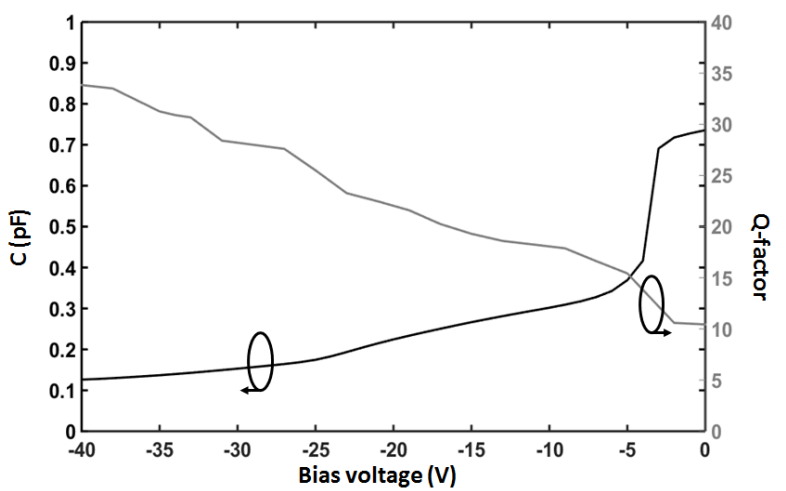

Fig. 2. Extracted varactor capacitance and $Q$-factor at $10 \mathrm{GHz}$ versus the tuning voltage of the $8 \times 50 \mu \mathrm{m}$ varactor.

The $8 \times 50 \mu \mathrm{m} \mathrm{GaN}$ varactor was characterized with two-port S-parameter measurements. The varactor capacitance $C(V)$ and quality factor $Q(V)$ versus the tuning voltage are extracted at $10 \mathrm{GHz}$ and are presented in Fig. 2. The $Q$-factor is extracted from a Deloach test structure based on the method in [11]. It can be seen that the $Q$-factor is poor for bias voltage $>-5 \mathrm{~V}$. Thus, the useful tuning range is defined to be between $-5 \mathrm{~V}$ to -40 V.
Below $-25 \mathrm{~V}$, the capacitance variation of the varactor decreases significantly.

\section{B. VCO measurements}

The designed VCO is characterized using an FSUP50 signalsource analyzer from Rohde \& Schwarz. The low-noise internal bias supplies of the FSUP are used for gate and drain biasings while the varactor voltage is biased with an external battery up to $50 \mathrm{~V}$.

First of all, the gate and drain bias voltages are swept from -2.2 to $0 \mathrm{~V}$ and from 6 to $10 \mathrm{~V}$, respectively while the bias voltage across the varactor is fixed at $-10 \mathrm{~V}$ in order to find the optimum bias point for lowest phase noise. It is found that a drain voltage of $6 \mathrm{~V}$ gives the best phase noise. Fig. 3 presents the drain current versus the gate voltage for different drain voltages. It is seen that the current of this VCO can be controlled well with the gate voltage.

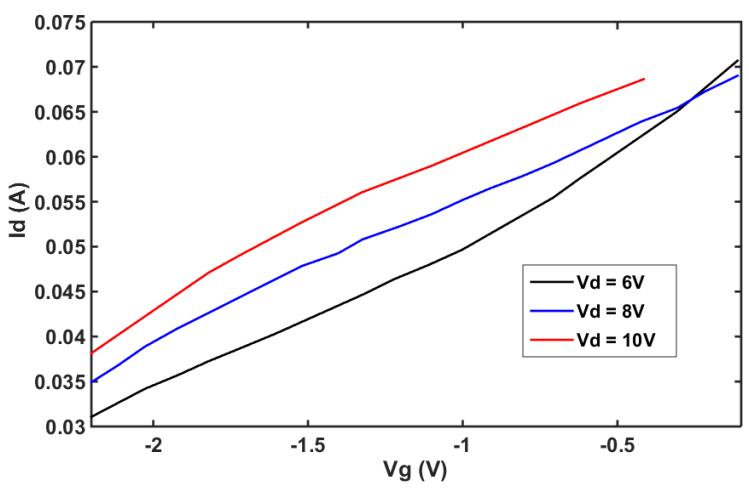

Fig. 3. Drain current versus gate voltage for different drain voltages.

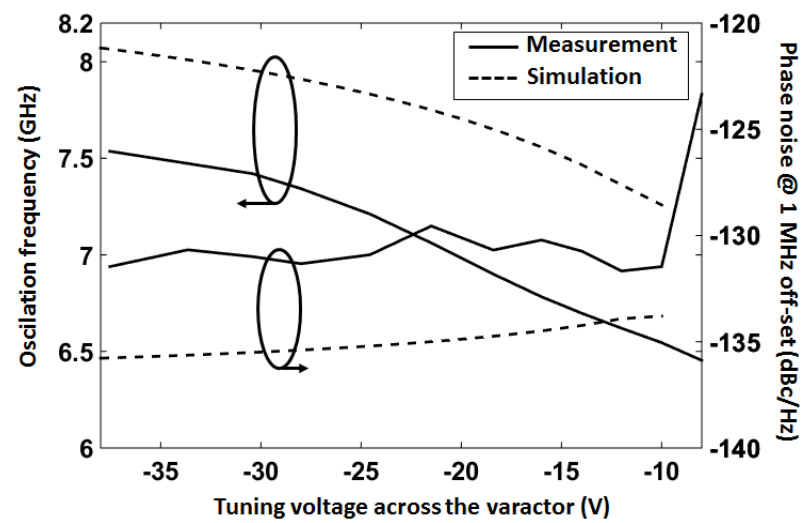

Fig. 4. Oscillation frequency and phase noise versus tuning voltage of the varactor.

Then, the gate and drain voltages are held constant at $-2 \mathrm{~V}$ and $6 \mathrm{~V}$, respectively, while tuning voltage across the varactor now is swept from $-8 \mathrm{~V}$ to $-38 \mathrm{~V}$. Fig. 4 shows the measured oscillation frequency versus varactor voltage compared to simulated result. The measured 
oscillation frequency is shifted down roughly $10 \%$ compared to simulation. Likely, this shift is due to parasitic resistance and coupling effects. Nevertheless, it can be seen that the tuning shape agrees well between measurement and simulation. The $\mathrm{VCO}$ has a tuning range about $15 \%(6.45-7.55 \mathrm{GHz})$.

Fig. 5 presents the measured and simulated output power versus tuning voltage of the varactor. The measured power is $1.5 \mathrm{dBm}$ higher than the simulated. Like the oscillation frequency, the shape of measured and simulated output power agree well. The output power varies from $-2 \mathrm{dBm}$ to $2 \mathrm{dBm}$.

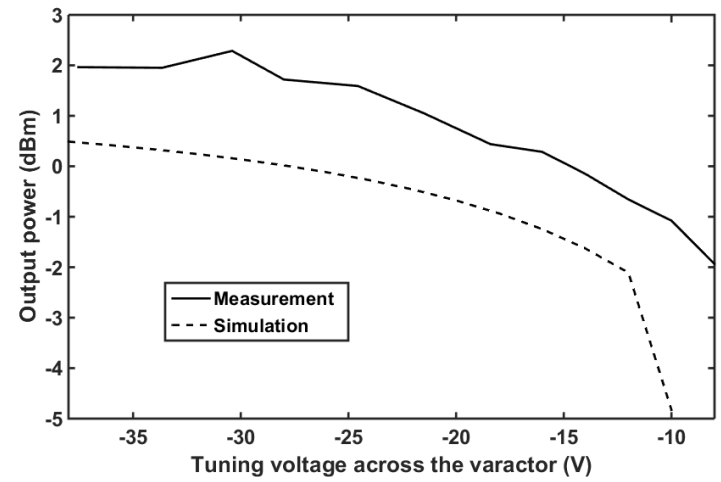

Fig. 5. Oscillator output power versus tuning voltage of the varactor.

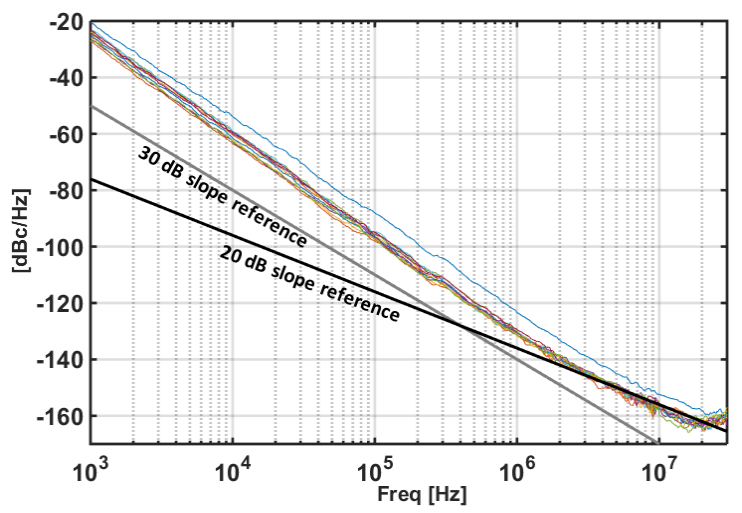

Fig. 6. Measured phase noise versus offset frequency with varactor voltage as parameter.

Fig. 6 shows the measured phase noise versus offset frequency with varactor tuning voltage as parameter. The lowest phase noise achieved is $-98 \mathrm{dBc} / \mathrm{Hz} @ 100 \mathrm{kHz}$ and $-132 \mathrm{dBc} / \mathrm{Hz} @ 1 \mathrm{MHz}$ offset frequencies, respectively, for a tuning voltage of $V_{v}=-28.5 \mathrm{~V}$. It can be seen that the GaN VCO is affected by up-conversion of low frequency noise. The corner frequency between $1 / f^{3}$ and $1 / f^{2}$ phase noise slope occurs about $2 \mathrm{MHz}$. From $1 \mathrm{kHz}$ to $10 \mathrm{kHz}$ offsets, the slope is almost $40 \mathrm{~dB} /$ decade which is a clear indication of generation-recombination $(G-R)$ noise appearance. Minimization of low frequency noise upconversion into phase noise is particularly important in GaN HEMT based VCO design.

The measured phase noise @ $1 \mathrm{MHz}$ offset versus varactor tuning voltage can be seen together with the oscillation frequency in Fig. 4. It is found that the measured result is about $2-4 \mathrm{~dB}$ higher than in the simulation. TABLE I shows a comparison of this VCO versus other fully integrated $\mathrm{GaN}$ and GaAs-InGaP VCOs reported in open literature. The phase noise performance @ $100 \mathrm{kHz}$ and @ $1 \mathrm{MHz}$ offsets of this VCO is better than other MMIC GaN VCOs, for comparable frequency. The $1 \mathrm{MHz}$ phase noise performance is also in the same level as good MMIC GaAs-InGaP HBT VCOs [9, 12].

\section{Phase noise simulation}

As mentioned above, Agilent ADS is used for the simulations. The phase noise simulation in ADS works fine when there is no flicker noise in the active device. To accurately predict the phase noise of a GaN HEMT oscillator, this work addresses the method proposed in [10]. To apply this method, a low-frequency noise (LFN) measurement and oscillator waveforms from $\mathrm{HB}$, i.e. transistor intrinsic current, tank resonance current, tank resonance voltage, are required. The LFN measurement setup used is presented in [13]. Fig. 7 shows the measured low frequency noise of the $2 \times 50 \mu \mathrm{m}$ HEMT and $1 / f$ noise model at $V_{d}=6 \mathrm{~V}$ and $V_{g}$ is swept from -3.8 to $-2.8 \mathrm{~V}$. The $1 / f$ noise model is defined as:

$$
\overline{i_{n}^{2}}=K_{f} \cdot \frac{I_{d}^{A f}}{f^{F f e}}
$$

where $I_{d}$ is the DC drain current, $A_{f}$ is the current dependency factor, $K_{f}$ is level fitting and $F_{f e}$ is the frequency dependent power factor. The extracted values are: $K_{f}=1.3 \mathrm{e}^{-10}, A_{f}=0.8, F_{f e}=1.3$.

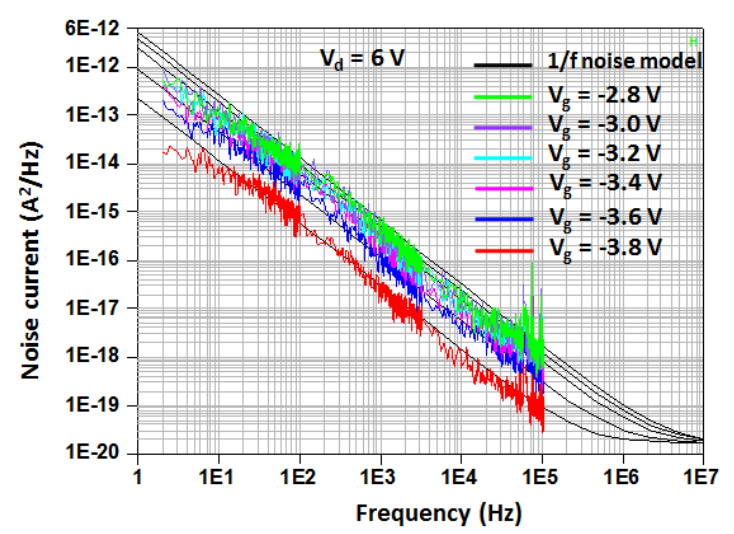

Fig. 7. Measured low frequency noise of the $2 \times 50 \mu \mathrm{m}$ HEMT and $1 / f$ noise model at $V_{d}=6 \mathrm{~V}, V_{g}$ is swept from -3.8 to $-2.8 \mathrm{~V}$. 
Fig. 8 shows measured phase noise versus offset frequency compared to the cyclostationary calculation and the phase noise simulation from ADS for a varactor voltage of $V_{v}=-28.5 \mathrm{~V}$. It can be seen that the calculated phase noise agrees very well with the measured result in the $1 / f^{3}$ region, while the ADS simulation is accurate in the $1 / f^{2}$ region.

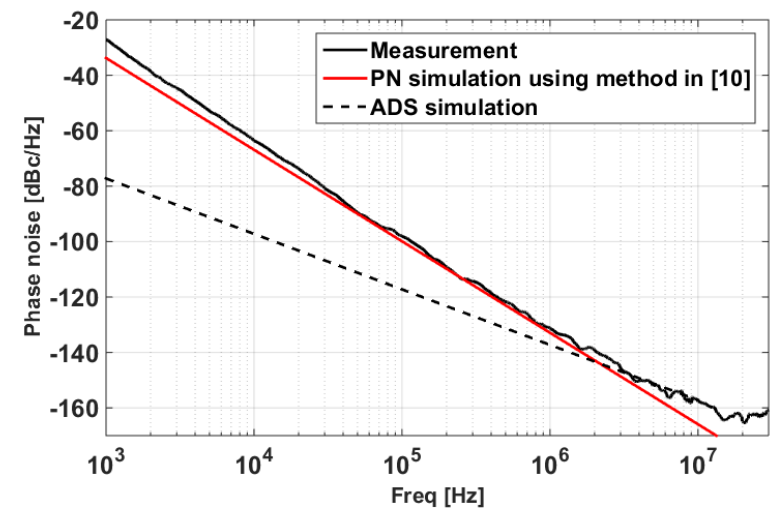

Fig. 8. Measured phase noise versus offset frequency compared to simulated phase noise using method in [10] and phase noise simulation in $\mathrm{ADS}$ at a varactor voltage of $V_{v}=-28.5 \mathrm{~V}$.

\section{CONCLUSION}

This paper reports on a medium tuning range (15\%) MMIC GaN HEMT based VCO with nearly constant output power, high tuning linearity, and a $1 \mathrm{MHz}$ offset phase noise that is better than state-of-the-art InGaP HBT VCOs with comparable tuning range. This work demonstrates the feasibility of wide tuning range and low phase noise VCOs in GaN HEMT MMIC technology.

\section{ACKNOWLEDGMENT}

This work was carried out in the GigaHertz Center in a joint project financed by the Swedish Governmental Agency of Innovation Systems (VINNOVA), Chalmers University of Technology, RUAG AB, and Ericsson AB.

\section{REFERENCES}

[1] S. Wu, Y.Bar-Ness, "OFDM in the presence of phase noise: consequences and solutions," IEEE Trans. on Communications, vol. 52, no. 11, pp. 1988-1996, 2004.

[2] Z. Q. Cheng, Y. Cai, J. Liu, Y. Zhou, K. M. Lau, K.J. Chen, "A Low Phase Noise X- Band MMIC VCO Using HighLinearity and Low-Noise Composite-Channel $\mathrm{Al}_{0.3} \mathrm{Ga}_{0.7} \mathrm{~N} / \mathrm{Al}_{0.05} \mathrm{Ga}_{0.95} \mathrm{~N} / \mathrm{GaN}$ HEMTs," IEEE Trans. Microwave Theory \& Tech., vol. 55, no. 1, pp. 23-29, 2007.

[3] V. Kaper, R. Thompson, T. Prunty, J. R. Shealy, "X-band AlGaN/GaN HEMT MMIC Voltage- Controlled
TABLE I

COMPARISON TO OTHER MMIC GAN AND INGAP HBT VCOS

\begin{tabular}{|l|c|c|c|c|c|c|c|}
\hline Technology & \multicolumn{2}{|c|}{$\begin{array}{c}\text { Frequency } \\
(\mathrm{GHz})\end{array}$} & $\begin{array}{c}\mathbf{P}_{\text {out }} \\
(\mathrm{dBm})\end{array}$ & $\begin{array}{c}\text { PN @ } \\
\mathbf{1 0 0} \mathbf{~ k H z} \\
(\mathrm{dBc} / \mathrm{Hz})\end{array}$ & $\begin{array}{c}\text { PN @ } \\
\mathbf{1} \mathbf{M H z} \\
(\mathrm{dBc} / \mathrm{Hz})\end{array}$ & Ref \\
& $\mathrm{f}_{\min }$ & $\mathrm{f}_{\max }$ & $\mathrm{BW}$ & & & & \\
\hline GaN HEMT & 9.11 & 9.55 & 0.4 & 3.3 & -82 & -110 & {$[2]$} \\
\hline GaN HEMT & 8.5 & 9.5 & 1.0 & 31.8 & -77 & -101 & {$[3]$} \\
\hline GaN HEMT & 6.5 & 7.5 & 1.0 & 17 & -81 & -110 & {$[4]$} \\
\hline GaN HEMT & 6.45 & 7.55 & $\mathbf{1 . 1}$ & $\mathbf{2}$ & -98 & -132 & $\begin{array}{c}\text { This } \\
\text { work }\end{array}$ \\
\hline InGaP HBT & 6.1 & 7.5 & 1.4 & -5 & -102 & -125 & {$[12]$} \\
\hline InGaP HBT & 8.0 & 9.7 & 1.7 & 7 & -106 & -128 & {$[9]$} \\
\hline
\end{tabular}

Oscillator," in Proceedings of $11^{\text {th }}$ Gallium Arsenide applications (GAAS) Symposium, pp. 45-48, 2003.

[4] C. Kong, H. Li, X. Chen, S. Jiang, J. Zhou, C. Chen, "A Monolithic AlGaN/GaN HEMT VCO Using BST ThinFilm Varactor," IEEE Trans. Microwave Theory \& Tech., vol. 60, no. 11, pp. 3413-3419, 2012.

[5] D. B. Leeson, "A simple model of feedback oscillator noise spectrum", in Proceedings of the IEEE 54(2), pp. 329-330, 1996.

[6] S. Lai, D. Kuylenstierna, M. Özen, M. Hörberg, N. Rorsman, I. Angelov, et al.,"Low Phase Noise GaN HEMT Oscillators With Excellent Figures of Merit," IEEE Microw. Compon. Lett., vol.24, pp. 412-414, 2014.

[7] M. Hörberg, D. Kuylenstierna, "Low phase noise powerefficient MMIC GaN HEMT Oscillator at $15 \mathrm{GHz}$ based on a Quasi-lumped on chip resonator," presented in IEEE MTT-S International Microwave Symposium Digest, 17-22 May, Phoenix, Arizona, 2015.

[8] H. Zirath, R. Kozhuharov, M. Ferndahl, "Balanced Colpittsoscillator MMICs designed for ultra-low phase noise," IEEE J. Solid-State Circuits, vol. 40, no. 10, pp. 2077-2086, 2005.

[9] D. Kuylenstierna, S. Lai, B. Mingquan, H. Zirath, "Design of Low-Phase-Noise Oscillators and Wideband VCOs in InGaP-HBT Technology," IEEE Trans. Microwave Theory \&Tech., vol. 60, pp. 3420-3430, 2012.

[10] S. Lai, D. Kuylenstierna, M. Hörberg, N. Rorsman, I. Angelov, K. Andersson, H. Zirath, "Accurate Phase noise Prediction for a balanced Colpitts GaN HEMT MMIC Oscillator," IEEE Trans. Microwave Theory \& Tech., vol. 61, no. 11, pp. 3916-3926, Nov., 2013.

[11] B. C. Deloach, "A new microwave measurement technique to characterize diodes and an 800-gc cutoff frequency varactor at zero volts bias," IEEE Trans. Microwave Theory \& Tech., vol. 12, no. 1, pp. 15-20, 1964.

[12] H. Zirath, "Low phase-noise balanced Colpitt InGaP-GaAs HBT VCOs with wide frequency tuning range and small VCO- gain variation," in IEEE Asia-Pacific Micro. Conf, Thailand, Dec. 11-14, 2007.

[13] T. N. T. Do, M. Hörberg, S. Lai, D. Kuylenstierna, "Low Frequency Noise Measurements - A Technology Benchmark with Target on Oscillator Applications," in Proceedings of 44th European Microwave (EuMW) Conf., Rome, Italy, pp. 468-471, Oct. 5-10, 2014. 